\title{
EXPONENTIALLY LONG TIME STABILITY NEAR AN EQUILIBRIUM POINT FOR NON-LINEARIZABLE ANALYTIC VECTOR FIELDS.
}

\author{
TIMOTEO CARLETTI
}

\begin{abstract}
We study the orbit behavior of a germ of an analytic vector field of $\left(\mathbb{C}^{n}, 0\right), n \geq 2$. We prove that if its linear part is semisimple, non-resonant and verifies a Bruno-like condition, then the origin is effectively stable: stable for finite but exponentially long times.
\end{abstract}

\section{INTRODUCTION}

Let us consider the germ of analytic vector field, $X_{F}=\sum_{1 \leq j \leq n} F_{j}(z) \frac{\partial}{\partial z_{j}}$, of $\left(\mathbb{C}^{n}, 0\right) n \geq 2$, whose components $\left(F_{j}\right)_{1 \leq j \leq n}$ are analytic functions vanishing at $0 \in \mathbb{C}^{n}$.

Let us consider the associated Ordinary Differential Equation:

$$
\frac{d z}{d t}=F(z)
$$

under the above assumptions $z(t ; 0)=0$ for all $t$ is an equilibrium solution ${ }^{1}$. We are interested in studying the stability of orbits of $X_{F}$ in a neighborhood of this equilibrium point.

We use the standard definition of stability (see Mo for an equilibrium solution: $z=0$ it is stable is the past and in the future if for any neighborhood $U$ of 0 there exists a neighborhood $\mathrm{V}$, containing the origin, s.t. $z\left(0 ; z_{0}\right) \in V$ implies $z\left(t ; z_{0}\right) \in U$ for all $t \in \mathbb{R}$.

In a coordinates system centered at the equilibrium point the $j$-th component of the vector field will take the form: $F_{j}(z)=(A z)_{j}+f_{j}(z)$, with $A$ a $n \times n$ complex matrix and $f_{j}$ analytic function such that $f_{j}(0)=D f_{j}(0)=0$, for all $1 \leq j \leq n$. Following the idea of Poincaré to study the orbit of (1.1) in a neighborhood of the origin, we will try to find an analytic change of coordinates, through an analytic diffeomorphisms $z \mapsto H(z)=w$ the linearization, s.t. in the new coordinates the vector field $X_{F}$ is conjugate to its linear part, $X_{A}=\sum(A z)_{j} \frac{\partial}{\partial z_{j}}: H^{*} X_{F} H^{-1}=X_{A}$. Hence equation (1.1) rewrites:

$$
\frac{d w}{d t}=A w
$$

Date: February 8, 2018.

1991 Mathematics Subject Classification. Primary 37C75, 34A25.

Key words and phrases. linearization vector field, Gevrey class, Bruno condition, effective stability, Nekhoroshev theorem.

${ }^{1}$ Here and throughout the paper by $z\left(t ; z_{0}\right)$ we mean the solution at time $t$ of 1.1 s.t. $z\left(0 ; z_{0}\right)=$ $z_{0}$. When the value of $z_{0}$ will not be relevant we'll just write $z(t)$. 
This change of coordinates must solve:

$$
A H(z)=D H(z) \cdot(A z+f(z)),
$$

and it is unique by assuming $D H(0)=\mathbb{I}$.

Clearly if the linear system (1.2) is stable and (1.1) is analytically linearizable, then also the latter is stable. It is a remarkable result that this condition is also necessary, as the following Theorem states:

Theorem 1.1 (Carathéodory-Cartan 1932). Necessary and sufficient condition for the stability of the solution $z=0$ of (1.1) for all real $t$ is that:

(1) A is diagonalizable with purely imaginary eigenvalues;

(2) there exists an holomorphic function $z=K(w)=w+\mathcal{O}\left(|w|^{2}\right), w \in \mathbb{C}^{n}$, which brings (1.1) into the linear system:

$$
\frac{d w}{d t}=A w .
$$

So let us assume $A$ to verify hypothesis of Theorem 1.1 let $\left(\omega_{j}\right)_{1 \leq j \leq n} \subset \mathbb{R}$ and $A=\operatorname{diag}\left(i \omega_{1}, \ldots, i \omega_{n}\right)$.

Then $A$ belongs to the Siegel domain ${ }^{2}$ : the origin is contained in the convex hull of the set of eigenvalues plotted as points in the complex plane (e segment in this case). This is the harder situation w.r.t. to the complementary case, Poincaré domain, because small divisors are involved: the existence of an analytic linearization is strictly related to the arithmetic property of approximation of the vector $\omega=\left(\omega_{1}, \ldots, \omega_{n}\right)$, with vectors with integer entries.

The first step is to assume $A$ to be non-resonant: $\alpha \cdot \omega \neq \omega_{j}$, for all $\alpha \in \mathbb{N}^{n}$ s.t. $|\alpha|=\alpha_{1}+\cdots+\alpha_{n} \geq 2$ and for all $j \in\{1, \ldots, n\}$. This ensures the existence of a formal change of variable which linearizes (1.1).

In $\left[\mathrm{Br}\right.$ author introduced the, today called, Bruno condition ${ }^{3}$ to characterize the rate of approximation of vectors of $\mathbb{R}^{n}$ with vectors of $\mathbb{Z}^{n}$ :

$$
\sum_{k \geq 0} \frac{\log \Omega^{-1}(k+1)}{2^{k}}<+\infty,
$$

where for all positive integers $k, \Omega(k)=\min \left\{\left|\alpha \cdot \omega-\omega_{j}\right|: j \in\{1, \ldots, n\}, \alpha \in\right.$ $\left.\mathbb{Z}^{n}, 0<|\alpha|<2^{k}\right\}$. From the result of Bruno $\underline{\mathrm{Br}}$ follows that if $A$ is non-resonant, diagonal with purely imaginary eigenvalues and verifies the above condition, then there exists an analytic linearization which brings (1.1) into (1.2).

Let us make a step backward and consider the following problem Ca2. Let $\mathcal{A}_{1} \subset \mathcal{A}_{2} \subset \mathbb{C}^{n}\left[\left[z_{1}, \ldots, z_{n}\right]\right]$ be two classes of formal power series closed w.r.t. to derivation and composition. Let $\hat{f} \in \mathcal{A}_{1}$ s.t. $\hat{f}=\sum_{|\alpha| \geq 2} f_{\alpha} z^{\alpha}$, let $A \in G L(n, \mathbb{C})$

\footnotetext{
${ }^{2}$ According to the classification of $\mathrm{Br}$ this case is Poincaré domain 1.d, but we prefer consider it as a Siegel case because the obstructions to the linearizability are very similar to those encountered in the Siegel domain.

${ }^{3}$ The Bruno condition can be rewritten using a general increasing sequence of integer numbers, $\left(p_{k}\right)_{k}$. In $\mathrm{Br}$ pag. 222, author proved that 1.4 is equivalent to:

$$
\sum_{k \geq 0} \frac{\log \hat{\Omega}^{-1}\left(p_{k+1}\right)}{p_{k}}<+\infty,
$$

where $\hat{\Omega}(p)=\min \left\{\left|\alpha \cdot \omega-\omega_{j}\right|: j \in\{1, \ldots, n\}, \alpha \in \mathbb{Z}^{n}, 0<|\alpha|<p,\right\}$.
} 
and consider the following (formal) ODE:

$$
\frac{d z}{d t}=A z+\hat{f}(z) .
$$

We say that (1.5) is linearizable in $\mathcal{A}_{2}$ if there exists $\hat{H} \in \mathcal{A}_{2}$, normalized with $\hat{H}=z+\mathcal{O}\left(|z|^{|\alpha|}\right),|\alpha| \geq 2$, s.t. formally we have:

$$
w=\hat{H}(z) \quad \text { and } \quad \frac{d w}{d t}=A w .
$$

If both $\mathcal{A}_{1}$ and $\mathcal{A}_{2}$ coincide with the ring of formal power series we already know that generically the problem has solution if and only if $A$ is non-resonant, which will be assumed from now. In the other cases of general algebras of formal power series, new arithmetical conditions on $A$ have to be imposed if we are in the Siegel domain. This case has been considered in details in Ca2 section 5, to which we refer for all details ${ }^{4}$. There author proved that the Bruno condition is still sufficient to linearize whenever $\mathcal{A}_{1}=\mathcal{A}_{2}$, otherwise new Bruno-like conditions are introduced, weaker than the original Bruno condition.

An interesting case is when $\mathcal{A}_{1}$ is the ring of convergent power series in some neighborhood of the origin, and $\mathcal{A}_{2}$ is the algebra of Gevrey-s, $s>0$, formal power series. Namely we are considering the Gevrey linearization of analytic vector fields.

Let $\hat{F}=\sum f_{\alpha} z^{\alpha},\left(f_{\alpha}\right)_{\alpha \in \mathbb{N}^{n}} \subset \mathbb{C}^{n}$ be a formal power series, then we say that it is Gevrey-s $\mathrm{Ba}, \mathrm{Ra}, s>0$, if there exist two positive constants $C_{1}, C_{2}$ such that:

$$
\left|f_{\alpha}\right| \leq C_{1} C_{2}^{-s|\alpha|}(|\alpha| !)^{s} \quad \forall \alpha \in \mathbb{N}^{n} .
$$

We denote the class of formal vector valued power series Gevrey-s by $\mathcal{C}_{s}$. It is closed w.r.t. derivation and composition.

In the Gevrey- $s$ case the arithmetical condition introduced in Ca2, called Bruno-s condition, $s>0$, for short $\mathcal{B}_{s}$, reads:

$$
\limsup _{|\alpha| \rightarrow+\infty}\left(2 \sum_{m=0}^{\kappa(\alpha)} \frac{\log \Omega^{-1}\left(p_{m+1}\right)}{p_{m}}-s \log |\alpha|\right)<+\infty,
$$

for some increasing sequence of positive integer $\left(p_{k}\right)_{k}$ and $\kappa(\alpha)$ is defined by $p_{\kappa(\alpha)} \leq$ $|\alpha|<p_{\kappa(\alpha)+1}$.

Remark 1.2. This definition recall the classical one of Bruno $[\mathrm{Br}$, where first one suppose the existence of a strictly increasing sequence of positive integer such that (1.7) holds, then one can prove (see $[\mathrm{Br}$ §IV page 222) that one can take an exponentially growing sequence, e.g. $p_{k}=2^{k}$. This holds also in our case, in fact we can prove that (1.7) is equivalent to:

$$
\limsup _{N \rightarrow+\infty}\left(\sum_{l=0}^{N} \frac{\log \Omega^{-1}\left(2^{l+1}\right)}{2^{l}}-s N 2 \log 2\right)<+\infty .
$$

A proof of this claim can be found in Ca1].

When $n=2$, under the above condition (non-resonance and Siegel domain), rescaling time by $-\omega_{2}$ (assuming $\omega_{2} \neq 0$ ), the ODE associated to the vector field

\footnotetext{
${ }^{4}$ See also $[\mathrm{CM}]$ where a similar problem for germs of diffeomorphisms of $(\mathbb{C}, 0)$ has been studied.
} 
can be rewritten as:

$$
\left\{\begin{array}{l}
\dot{z}_{1}=\omega z_{1}+\text { h.o.t. } \\
\dot{z}_{2}=-z_{2}+\text { h.o.t. }
\end{array},\right.
$$

where $\omega=-\omega_{1} / \omega_{2} \in(\mathbb{R} \backslash \mathbb{Q})^{+}$and high order terms means $\mathcal{O}\left(|z|^{|\alpha|}\right)$ with $|\alpha| \geq 2$, namely only the ratio of the eigenvalues enters. Then the Bruno- $s$ condition can be slightly weakened (see [CM]):

$$
\limsup _{n \rightarrow+\infty}\left(\sum_{j=0}^{k(n)} \frac{\log q_{j+1}}{q_{j}}-s \log n\right)<+\infty,
$$

where $k(n)$ is defined by $q_{k(n)} \leq n<q_{k(n)+1}$ and $\left(q_{n}\right)_{n}$ are the denominators of the convergents [HW] to $\omega$.

We remark that in both cases the new conditions are weaker than Bruno's condition, which is recovered when $s=0$. When $n=2$ we prove that the set $\bigcup_{s} \mathcal{B}_{s}$ is $\operatorname{PSL}(2, \mathbb{Z})$-invariant (see remark 3.1 ).

The main result of [Ca2] in the case of Gevrey- $s$ classes reads:

Theorem 1.3 (Gevrey-s linearization). Let $\omega_{1}, \ldots, \omega_{n}$ be real numbers and $A=$ $\operatorname{diag}\left(i \omega_{1}, \ldots, i \omega_{n}\right)$; let $D_{1}=\left\{z \in \mathbb{C}^{n}:\left|z_{i}\right|<1,1 \leq i \leq n\right\}$ be the isotropic polydisk of radius 1 and let $F: D_{1} \rightarrow \mathbb{C}^{n}$ be an analytic function, such that $F(z)=A z+f(z)$, with $f(0)=D f(0)=0$. If $A$ is non-resonant and verifies a Bruno-s, $s>0$, condition (1.7) (or condition (1.9) if $n=2$ ), then there exists a formal Gevrey-s linearization $\hat{H}$.

The aim of this paper is to show that the Gevrey character of the formal linearization can give information concerning the dynamics of the analytic vector field. Let $F(z)=A z+f(z)$ verify hypotheses of Theorem 1.3 assume moreover $X_{F}$ not to be analytically linearizable. We will show that even if there is not a Stable domain, where the dynamics of $X_{F}$ is conjugate to the dynamics of its linear part, we have an open neighborhood of the origin which "behaves as a Stable domain" for the flow of $X_{F}$ for finite but long time, which results exponentially long: the effective stability GFGS, GP of the equilibrium solution.

In the case of analytic linearization, $\left|H_{j}(z)\right|, j=1, \ldots, n$, is constant along the orbits, namely it is a first integral and the flow of (1.1) is bounded for all $t$ and sufficiently small $\left|z_{0}\right|$.

We will prove that any non-zero $z_{0}$ belonging to a polydisk of sufficiently small radius $r>0$, can be followed up to a time $T=\mathcal{O}\left(\exp \left\{r^{-1 / s}\right\}\right)$, being $s>0$ the Gevrey exponent of the formal linearization, and we can find an almost first integral: a function which varies by a quantity of order $r$ during this interval of time. More precisely we prove the following

Theorem 1.4. Let $n \in \mathbb{N}, n \geq 2$. Given real $\omega_{1}, \ldots, \omega_{n}$ consider $A=\operatorname{diag}\left(i \omega_{1}, \ldots, i \omega_{n}\right)$; let $F: D_{1} \rightarrow \mathbb{C}^{n}$ be an analytic function, such that $F(z)=A z+f(z)$, with $f(0)=D f(0)=0$. If $A$ is non-resonant and verifies a Bruno-s, $s>0$, condition (1.7) (or (1.9) if $n=2$ ), then for all sufficiently small $0<r_{* *}<1$, there exist positive constants $A_{* *}, B_{* *}, C_{* *}$ such that for all $0<\left|z_{0}\right|<r_{* *} / 2$, the solutions $z\left(t ; z_{0}\right)$ are well defined and verify $\left|z\left(t ; z_{0}\right)\right| \leq C_{* *} r_{* *}$, for all $|t| \leq T_{*}=$ $A_{* *}^{-1} \exp \left\{B_{* *}\left(r_{* *} /\left|z_{0}\right|\right)^{1 / s}\right\}$. 
We want to stress here that when $s \rightarrow 0$ the stability time goes to infinity, because the critical exponent of stability time is $1 / s$ : namely one get stability. At the same time the Bruno-s condition "tends" to the classical Bruno condition, which is a sufficient condition to ensure analytic linearizability and hence stability under our assumptions. So we recover the classical stability result as limit of longer and longer effective stability times.

Results similar to Theorem 1.4 have been obtained in GFGS for hamiltonian vector fields and in GP for real reversible systems of coupled harmonic oscillators. In both papers effective stability is proved by assuming the linear part of the vector field to verify some Diophantine condition ${ }^{5} C D(\gamma, \tau)$, for some $\gamma>0$ and $\tau>n-1$, and the critical exponent of stability time is $1 / \tau$. In our result, too, the critical exponent of stability time depends on some arithmetical property of the linear part of the vector field but in a more general way in fact we assume $A \in \mathcal{B}_{s} \supset C D(\gamma, \tau)$, for all $\gamma>0$ and $\tau \geq n-1$.

The second remark is that in GFGS GP effective stability is obtained using some partial normal form, then working on it and using the Poincaré summation at the smallest term (see Lemma 2.2), the proof is done. Here the method used is completely different: we first linearize formally the system and then using properties of the formal linearization we conclude still using the Poincaré summation at the smallest term. This method introduce also our main drawback: we must assume $A$ to be non-resonant (to linearize) and this prevents us from considering real vector fields and hamiltonian ones, where an "intrinsic" resonance is present.

In section 3 we discuss the relation between the Bruno- $s$ condition and other arithmetical conditions.

\section{Proof of the main Theorem}

In this part we will prove our main result, Theorem 1.4 The proof will be divided into three steps: first we use the Gevrey- $s$ character of the formal linearization $\hat{H}$, given by Theorem 1.3 to find an approximate solution of the conjugacy equation (1.3) up to a (exponentially) small correction (paragraph 2.1); then we prove a Lemma allowing us to control how the small error introduced in the solution propagates (paragraph 2.2). Finally we collect all the informations to conclude the proof (paragraph [2.3).

2.1. Determination of an approximate solution. Let $F$ verifies hypotheses of Theorem 1.4 and let us consider the first order differential equation in $\mathbb{C}^{n}, n \geq 2$ :

$$
\frac{d z}{d t}=F(z) .
$$

By Theorem 1.3 this system can be put in linear form by a formal power series $\hat{H}$ which belongs to $\mathcal{C}_{s}$ and it solves (formally):

$$
\frac{d}{d t} \hat{H}(z)=A \hat{H}(z)
$$

we observe that one can choose $\hat{H}(z)=z+\mathcal{O}\left(|z|^{2}\right)$.

\footnotetext{
${ }^{5} \mathrm{~A}$ vector $\omega \in \mathbb{R}^{n}$ belongs to $C D(\gamma, \tau)$ if there exists $\gamma>0$ and $\tau>n-1$ such that for all $\alpha \in \mathbb{N}^{n}$ and all $j \in\{1, \ldots, n\}$ one has: $\left|\alpha \cdot \omega-\omega_{j}\right| \geq \gamma|\alpha|^{-\tau}$. Let $A=\operatorname{diag}\left(\omega_{1}, \ldots, \omega_{n}\right)$, then $A$ verifies a Diophantine condition if $\omega$ does.
} 
Since $\hat{H}=\sum h_{\alpha} z^{\alpha} \in \mathcal{C}_{s}$, there exist positive constants $A_{1}$ and $B_{1}$ such that

$$
\left|h_{\alpha}\right| \leq A_{1} B_{1}^{-s|\alpha|}(|\alpha| !)^{s} \quad \forall|\alpha| \geq 1 .
$$

For any positive integer $N$ we consider the vectorial polynomial, sum of homogeneous vector monomials of degree $1 \leq l \leq N$, defined by: $\mathcal{H}_{N}(z)=\sum_{l=1}^{N} \sum_{|\alpha|=l} h_{\alpha} z^{\alpha}$ and the Remainder Function:

$$
\mathcal{R}_{N}(z)=D \mathcal{H}_{N}(z) \cdot F(z)-A \mathcal{H}_{N}(z) .
$$

Clearly $\mathcal{H}_{N}(z)$ doesn't solve the linearization problem, but:

$$
\frac{d}{d t} \mathcal{H}_{N}(z)=A \mathcal{H}_{N}(z)+\mathcal{R}_{N}(z)
$$

hence the remainder function gives the difference from the true solution and the approximate one.

The following Proposition collects some properties of the remainder function.

Proposition 2.1. Let $\mathcal{R}_{N}(z)$ be the remainder function defined in (2.4) and let $\alpha \in \mathbb{N}^{n}$, then:

1) $\partial_{z}^{\alpha} \mathcal{R}_{N}(0)=0$ if $|\alpha| \leq N$.

2) For all $0<r<1$ there exist positive constants $A_{2}$ and $B_{2}$ such that if $|\alpha| \geq N+1$, then:

$$
\left|\frac{1}{\alpha !} \partial_{z}^{\alpha} \mathcal{R}_{N}(0)\right| \leq A_{2} r^{-|\alpha|} B_{2}^{-s N}(N !)^{s} .
$$

3) For all $0<r<1$ and $|z|<r / 4$ there exist positive constants $A_{3}, B_{3}$ such that:

$$
\left|\mathcal{R}_{N}(z)\right| \leq A_{3} B_{3}^{-s N}(N !)^{s}\left(\frac{|z|}{r}\right)^{N+1} .
$$

Where we used the compact notation $\frac{1}{\alpha !} \partial_{z}^{\alpha}=\frac{1}{\alpha_{1} ! \ldots \alpha_{n} !} \frac{\partial^{|\alpha|}}{\partial_{z_{1}}^{\alpha} \ldots \partial_{z_{n}}^{\alpha_{n}}}$.

Proof. Statement 1) is an immediate consequence of the definition of $\mathcal{R}_{N}$.

To prove 2) we observe that $\mathcal{R}_{N}(z)$ is an analytic function on $D_{1}$, being obtained with product of analytic functions, then one gets by Cauchy's estimates for all $0<r<1$ and for all $|\alpha| \geq N+1$ :

$$
\left|\frac{1}{\alpha !} \partial_{z}^{\alpha} \mathcal{R}_{N}(0)\right| \leq \frac{1}{(2 \pi)^{n}} \frac{1}{r^{|\alpha|+1}} \max _{|z|=r}\left|D \mathcal{H}_{N} \cdot F(z)\right|,
$$

because $\partial^{\alpha} \mathcal{H}_{N}=0$ for $|\alpha| \geq N+1$. Recalling the Gevrey estimate (2.3) for $\mathcal{H}_{N}$ and the analyticity of $F$ we obtain:

$$
\left|\frac{1}{\alpha !} \partial_{z}^{\alpha} \mathcal{R}_{N}(0)\right| \leq A_{2} B_{2}^{-s N}(N !)^{s} r^{-|\alpha|},
$$

for some positive constants $A_{2}$ and $B_{2}$ depending on the previous constants, on the dimension $n$ and on $F$.

To prove 3) let us write the Taylor series $\mathcal{R}_{N}(z)=\sum_{|\alpha| \geq N+1} \frac{1}{\alpha !} \partial_{z}^{\alpha} \mathcal{R}_{N}(0) z^{\alpha}$ : the bound on derivatives (2.8) implies the estimate (2.6) for all $|z|<r / 4$ and for some positive constants $A_{3}$ and $B_{3}$.

The bound (2.6) on $\mathcal{R}_{N}(z)$ depends on the positive integer $N$, so we can determine the value of $N$ for which the right hand side of (2.6) attains its minimum, that's Poincaré's idea of summation at the smallest term. 
Lemma 2.2 (Summation at the smallest term). Let $\mathcal{R}_{N}(z)$ defined as before and let $0<r_{*}<1 / 4$ then there exist positive constants $A_{4}, B_{4}$ such that for all $0<|z|<r_{*}$ we have:

$$
\left|\mathcal{R}_{\bar{N}}(z)\right| \leq A_{4} \exp \left\{-B_{4}\left(\frac{r_{*}}{|z|}\right)^{1 / s}\right\}
$$

where $\bar{N}=\left\lfloor B_{4}\left(r_{*} /|z|\right)^{1 / s}\right\rfloor$ and $\lfloor x\rfloor$ denotes the integer part of $x \in \mathbb{R}$.

Proof. Let us fix $0<r_{*}<1 / 4$, then for $0<|z|<r_{*}$ by Stirling formula we obtain:

$$
\left|\mathcal{R}_{N}(z)\right| \leq A_{4}\left(N B_{3}^{-1}\left(|z| / r_{*}\right)^{1 / s}\right)^{N s} e^{-s N},
$$

for some positive constant $A_{4}$. The right hand side of (2.10) attains its minimum at $\bar{N}=B_{3}\left(r_{*} /|z|\right)^{1 / s}$, evaluating the value of this minimum we get (2.9) with $B_{4}=B_{3}$.

2.2. Control of the "errors". Let us define $\mathcal{H}(z)=\mathcal{H}_{\bar{N}}(z)$ and $\mathcal{R}(z)=\mathcal{R}_{\bar{N}}(z)$, being $\bar{N}$ the "optimal value" obtained in Lemma 2.2 We remark that $\mathcal{H}(z)$ doesn't solve (2.2) but the "error", $\mathcal{R}(z)$, can be made very small: exponentially small. We will prove that for initial conditions in a sufficiently small disk, one can follow the flows for an exponential long time without leaving a disk comparable size.

Lemma 2.3 (Control the flow). Let $a, b, \alpha$ and $R$ be positive real numbers. Let $T=R a^{-1} e^{b /(2 R)^{\alpha}}$ and let us consider the Cauchy problem:

$$
\left\{\begin{array}{l}
\frac{d}{d t} x(t)=a e^{-b / x^{\alpha}} \\
x(0)=R .
\end{array}\right.
$$

Then $0<x(t)<2 R$ for all $|t|<T$.

Proof. Let us write the Cauchy problem in integral form:

$$
x(t)=R+\int_{0}^{t} a e^{-b /(x(s))^{\alpha}} d s
$$

$x(t)$ is trivially monotonically increasing, hence the same holds for the function $t \mapsto a e^{-b /(x(t))^{\alpha}}$. Let us suppose that there exists $0<t_{0}<T$, for which $x\left(t_{0}\right)=2 R$; then

$$
2 R=x\left(t_{0}\right)=R+\int_{0}^{t_{0}} a e^{-b /(x(s))^{\alpha}} d s<R+t_{0} a e^{-b /\left(x\left(t_{0}\right)\right)^{\alpha}},
$$

namely $t_{0}>R a^{-1} e^{b /(2 R)^{\alpha}}=T$, which gives a contradiction. Hence either $x\left(t_{0}\right)>$ $2 R$ for all $0<t<T$ or $x\left(t_{0}\right)<2 R$, but the first case have to be excluded because $x(0)=R<2 R$.

The case $t<0$ can be handle in a similar way by showing that $t \mapsto x(t)$ doesn't decrease too much.

Let $r_{*}$ as in Lemma2.2 define $\rho(z)=|\mathcal{H}(z)|$ for all $0<|z|<r_{*}$, then Lemma2.2 admits the following Corollary, which allows us to control the evolution of $\rho(z)$.

Corollary 2.4. Let $0<r_{*}<1 / 4$, then there exists $0<r_{* *} \leq r_{*}$ and positive constants $A_{*}, B_{*}$ such that for all $0<|z|<r_{* *}$ we have:

$$
\frac{d}{d t} \rho(z) \leq A_{*} \exp \left\{-B_{*}\left(\frac{r_{*}}{\rho(z)}\right)^{1 / s}\right\} .
$$


Proof. Let $j \in\{1, \ldots, n\}$ and let us consider the time evolution of $\left|\mathcal{H}_{j}(z(t))\right|$. If $\mathcal{H}$ was a solution this would be a constant of motion, this is not the case but its evolution is nevertheless very slow. In fact thanks to (2.5) we get:

$$
\frac{d}{d t}\left|\mathcal{H}_{j}(z)\right| \leq\left|\mathcal{R}_{j}(z)\right|
$$

hence a similar statement holds for $\rho(z)=|\mathcal{H}(z)|=\max _{1 \leq j \leq n}\left|\mathcal{H}_{j}(z)\right|$.

We want now to express the exponential smallness of $|\mathcal{R}(z)|$ in terms of $\rho(z)$ instead of $|z| . \mathcal{H}(z)$ is tangent to the identity close to zero and then locally invertible. The inverse is still tangent to the identity, vanishing at zero and analytic in a neighborhood of the origin, then sufficiently close to the origin we have $\left|\mathcal{H}^{-1}(w)\right| \leq C|w|$, for some $C>0$. Finally we can take $|z|$ sufficiently small, say $|z|<r_{* *}$ for some $0<r_{* *} \leq r_{*}$, s.t. $|z| \leq C|\mathcal{H}(z)|<r_{*}$, hence:

$$
|\mathcal{R}(z)| \leq A_{4} \exp \left\{-B_{4}\left(\frac{r_{*}}{|z|}\right)^{1 / s}\right\} \leq A_{4} \exp \left\{-B_{4} C^{-1 / s}\left(\frac{r_{*}}{\rho(z)}\right)^{1 / s}\right\},
$$

and the claim follows with $A_{*}=A_{4}$ and $B_{*}=B_{4} C^{-1 / s}$.

2.3. End of the proof. We are now able to conclude the proof of the main Theorem 1.4 Take any $0<\left|z_{0}\right|<r_{* *} / 2$ and let $\rho_{0}=\left|\mathcal{H}\left(z\left(0 ; z_{0}\right)\right)\right|$, then there exists a positive constant $C_{1}$ s.t. $\rho_{0} \leq C_{1}\left|z_{0}\right|$. By Corollary 2.4 we have

$$
\frac{d}{d t} \rho\left(z\left(t ; z_{0}\right)\right) \leq A_{*} \exp \left\{-B_{*}\left(r_{*} / \rho\left(z\left(t ; z_{0}\right)\right)\right)^{1 / s}\right\} .
$$

Let us call $R=\rho_{0}, a=A_{*}, b=B_{*} r_{*}^{1 / s}$ and $\alpha=1 / s$ then we can apply Lemma 2.3. to conclude:

$$
\rho\left(z\left(t ; z_{0}\right)\right) \leq 2 \rho_{0}<C_{1} r_{* *} \quad \forall|t| \leq T_{*}=\rho_{0} A_{*}^{-1} \exp \left\{B_{*}\left(\frac{r_{*}}{2 \rho_{0}}\right)^{1 / s}\right\} .
$$

This implies that $\mathcal{H}\left(z\left(t ; z_{0}\right)\right)$ is well defined in this interval of time, it is not constant and $\left|\mathcal{H}\left(z\left(t ; z_{0}\right)\right)\right| \leq<3 C_{1} r_{* *}$. Recalling that $\mathcal{H}(z)$ is tangent to the identity close to zero, we have $|z| \leq C_{3}|\mathcal{H}(z)|$ for some positive $C_{3}$.

Then setting $A_{* *}=2 A_{*} r_{* *}^{-1}, B_{* *}=B_{*}\left(r_{*} /\left(2 r_{* *}\right)\right)^{1 / s}$ and $C_{* *}=C_{1} C_{3}$, we get:

$$
\left|z\left(t ; z_{0}\right)\right| \leq C_{* *} r_{* *},
$$

for all $|t| \leq A_{* *}^{-1} \exp \left\{B_{* *}\left(\frac{r_{* *}}{\left|z_{0}\right|}\right)^{1 / s}\right\}$.

\section{ARithmetical CONDitions}

In this paper we proved that any analytic germs of vector field of $\left(\mathbb{C}^{n}, 0\right)$ with diagonal, non-resonant linear part has an effective stability domain, i.e. stable up to finite but "long times", close to the stationary point, provided the linear part verifies an arithmetical Bruno-like condition depending on a parameter $s>0$, which in the case of 2 dimensional vector fields can be put in the form:

$$
\limsup _{n \rightarrow+\infty}\left(\sum_{j=0}^{k(n)} \frac{\log q_{j+1}}{q_{j}}-s \log n\right)<+\infty .
$$


Remark 3.1 (Invariance of $\bigcup_{s>0} \mathcal{B}_{s}, n=1$ under the action of $\operatorname{PSL}(2, \mathbb{Z})$ ). The continued fraction development $\mathrm{HW}$, MMY of an irrational number $\omega$ gives us the sequences: $\left(a_{k}\right)_{k \geq 0}$ and $\left(\omega_{k}\right)_{k \geq 0}$. Then we introduce $\left(\beta_{k}\right)_{k \geq-1}$ defined by $\beta_{-1}=1$ and for all integer $k \geq 0: \beta_{k}=\prod_{j=0}^{k} \omega_{k}$, which verifies : $1 / 2<\beta_{k} q_{k+1}<1$ and $q_{n} \beta_{n-1}+q_{n-1} \beta_{n}=1$, where $q_{k}$ 's are the denominators of the continued fraction development of $\omega$. We claim that condition Bruno-s (1.9) is equivalent to the following one:

$$
\limsup _{k \rightarrow+\infty}\left(\sum_{j=0}^{k} \beta_{j-1} \log \omega_{j}^{-1}+s \log \beta_{k-1}\right)<+\infty .
$$

This can be proved by using the relations between $\beta_{l}$ and $q_{l}$, to obtain the bound, for all integer $k>0$ :

$\left|\sum_{l=0}^{k}\left(\beta_{l-1} \log \omega_{l}+\frac{\log q_{l+1}}{q_{l}}\right)\right| \leq \sum_{l=0}^{k}\left|\beta_{l-1} \log \beta_{l} q_{l+1}\right|+\left|\beta_{l-1} \log \beta_{l-1}\right|+\left|\frac{q_{l-1}}{q_{l}} \beta_{l} \log q_{l+1}\right| \leq 18$,

where we used the convergence of series $\sum q_{l}^{-1}$ and $\sum q_{l}^{-1} \log q_{l}$ (see [MMY] page 272).

To prove the invariance of $\bigcup_{s} \mathcal{B}_{s}$ under the action of $P S L(2, \mathbb{Z})$, is enough to consider its generators: $T \omega=\omega+1$ and $S \omega=1 / \omega$. For any irrational $\omega, T$ acts trivially being $\beta_{k}(T \omega)=\beta_{k}(\omega)$ for all $k$, whereas for $S$ we have $\beta_{k}(\omega)=\omega \beta_{k-1}(S \omega)$ for all $k \geq 1$. Let $\omega$ be an irrational and let $\omega^{\prime}=\omega^{-1}$, let us also denote with a ' quantities given by the continued fraction algorithm applied to $\omega^{\prime}$, then using (3.1) one can prove:

$\omega_{0}\left(\sum_{j=0}^{k} \beta_{j-1}^{\prime} \log \omega_{j}^{\prime-1}+s \omega_{0}^{-1} \log \beta_{k-1}^{\prime}\right)=C(\omega, s)+\sum_{j=0}^{k+1} \beta_{j-1} \log \omega_{j}^{-1}+s \log \beta_{k}$,

where $C(\omega, s)=\omega_{0}\left(\log \omega_{1}^{-1}-s \log \omega_{0}\right)+\sum_{l=0}^{1} \beta_{l-1} \log \omega_{l}^{-1}$, from which the claim follows.

Let us consider a slightly stronger version of the Bruno-s condition: $\omega \in(0,1) \backslash \mathbb{Q}$ belongs to $\tilde{\mathcal{B}}_{s}$ if:

$$
\lim _{n \rightarrow+\infty}\left(\sum_{l=0}^{k} \frac{\log q_{l+1}}{q_{l}}-s \log q_{k}\right)<+\infty,
$$

where $\left(q_{n}\right)_{n}$ are the convergents to $\omega$, and let us introduce a second arithmetical condition denoted by $\mathcal{B}_{s}^{\prime}$ to be the set of irrational numbers whose convergents verify:

$$
\lim _{k \rightarrow+\infty} \frac{\log q_{k+1}}{q_{k} \log q_{k}}=s .
$$

We state without proof the following proposition, and we refer to Ca1, to all details:

Proposition 3.2. Let $s>0$ and let $\omega \in(0,1) \cap \tilde{\mathcal{B}}_{s}$. Then if $\omega$ is not a Bruno number then $\omega \in \mathcal{B}_{s}^{\prime}$, otherwise $\omega \in \mathcal{B}_{0}^{\prime}$.

Therefore if $\omega \in \tilde{\mathcal{B}}_{s} \backslash \mathcal{B}$ then the denominators of the convergent to $\omega$ can grow like a factorial, more precisely, $q_{k+1}=\mathcal{O}\left(\left(q_{k} !\right)^{s}\right)$, is allowed. 


\section{REFERENCES}

[Ba] W. Balser: From Divergent Power Series to Analytic Functions. Theory and Applications of Multisummable Power Series, Lectures Notes in Mathematics, 1582, Springer, (1994).

[Br] A.D. Bruno: Analytical form of differential equations, Transactions Moscow Math.Soc. 25, (1971), pp. 131-288.

$[\mathrm{CM}] \quad$ T. Carletti and S. Marmi:Linearization of analytic and non-analytic germs of diffeomorphisms of ( $\mathbb{C}, 0)$, Bull. Soc. Math. Française, 128, (2000), pp. 69-85.

[Ca1] T.Carletti: Exponentially long time stability for non-linearizable analytic germs of $\left(\mathbb{C}^{n}, 0\right)$., in press to Ann. Inst. Fourier, (2004).

[Ca2] T. Carletti: The Lagrange inversion formula on non-Archimedean fields. Nonanalytical form of differential and finite difference equations, DCDS series A, Vol. 9, N. 4, (2003), pp. 835-858.

[GFGS] A. Giorgilli, A. Fontich, L. Galgani and C. Simó: Effective stability for a Hamiltonian system near an elliptic equilibrium point, with an application to the restricted three body problem, J. of Differential Equations, 77, (1989), pp. 167-198.

[GP] A. Giorgilli and A. Posilicano: Estimates for normal forms of differential equations near an equilibrium point, J. of Appl. Math. and Phys. (ZAMP), 39, (1989), pp. 713732 .

[HW] G.H.Hardy and E.M.Wright: An introduction to the theory of numbers, $5^{\text {th }}$ edition Oxford Univ. Press, 1979.

[MMY] S.Marmi, P.Moussa and J.-C.Yoccoz: The Brjuno functions and their regularity properties, Communications in Mathematical Physics 186, (1997), pp. 265 - 293.

[Mo] J. Moser: Stable and random motions in dynamical systems, Annals of Math. Stud., 77, Princeton Univ. Press, (1977).

[Ra] J.-P. Ramis: Séries divergentes et Théorie asymptotiques, Publ. Journées X-UPS, (1991), pp. 1-67.

(Timoteo Carletti) Scuola Normale Superiore piazza dei Cavalieri 7, 56126 Pisa, Italia

E-mail address, Timoteo Carletti: t.carletti@sns.it 